\title{
Intermediate asymptotic behavior of the stress and damage fields in the vicinity of the mixed-mode crack tip under creep regime
}

\author{
L. Stepanova ${ }^{1}$, E. Mironova ${ }^{1}$

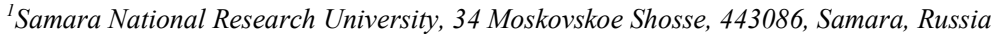

\begin{abstract}
The creep crack problems in damaged materials under mixed mode loading (Mode I and Mode II loading) in the framework of creep-damage coupled formulation are considered. The class of the self-similar solutions to the plane creep crack problems in a damaged medium under mixed-mode loading is given. With the similarity variable and the self-similar representation of the solution for a power-law creeping material and the Kachanov- Rabotnov power-law damage evolution equation the near crack-tip stresses, creep strain rates and continuity distributions for plane stress and plane strain conditions are obtained. The similarity solutions are based on the hypothesis of the existence of the completely damaged zone near the crack tip. It is shown that the asymptotic analysis of the near crack-tip fields gives rise to the nonlinear eigenvalue problems. The technique permitting to find all the eigenvalues numerically is proposed and numerical solutions of the nonlinear eigenvalue problems arising from the mixed-mode crack problems in a power-law medium under plane stress conditions are obtained. Using the approach developed the eigenvalues different from the eigenvalues corresponding to the Hutchinson-Rice-Rosengren (HRR) problem are found. The angular distributions of the stress and the continuity fields are selected as the crack tip fields of interest. Having obtained the eigenspectra and eigensolutions the geometry of the completely damaged zone in the vicinity of the crack tip is found for all values of the mixity parameter.
\end{abstract}

Keywords: damage parameter; continuity parameter; stress-strain fields near the crack tip; mixed-mode loading; asymptotic solution; similarity variable, self-similar solution; creep-damage coupling; nonlinear eigenvalue problems; eigenvalue spectrum

\section{Introduction}

Important advances in creep damage models for crack growth analysis have been made in the last two decades as scientists and engineers strive to imbue continuum-based models with more realistic details at microstructure damage mechanisms in the creep process [1-16]. Such damage models can be also found in [17-20]. Knowledge of stress, strain and displacement fields in the vicinity of the crack tip under mixed-mode loading conditions is important for the justification of fracture mechanics criteria and has attracted considerable attention nowadays [2-16]. Asymptotic analysis of the mechanical fields in front of stationary and propagating cracks facilitate the understanding of the mechanical and physical state in front of crack tips and they enable prediction of crack growth and failure. Furthermore, together with the stress, strain and displacement fields in the vicinity of the crack tip the damage distribution around the crack tip is a question of special attention $[4,6,9,19,20]$. Damage field around a crack tip essentially affects the surrounding stress field, and hence governs the crack extension behavior in the material. This effect of the damage field is an important problem either in the discussion of stability and convergence in crack extension analysis. So far mainly crack problems for the pure opening mode I at symmetrical loading have been thoroughly treated [6], [14]. The corresponding fracture criteria have been obtained on the assumption that the crack continues to extend along its original line (two-dimensional case) or plane (three-dimensional case) in a straightforward manner on the ligament. Nowadays the analysis of mixed-mode loading of cracked structures in nonlinear materials is of particular interest. In engineering practice, there are plenty of examples and reasons leading to mixed-mode loading of cracked structures when mode I is superimposed by mode II and/or III, the symmetry (or antisymmetry) is violated and the situation is related to mixed-mode loading [7]. The type of loading on a structure (tension, shear, bending, torsion) can also change during service. For a crack this results in an alteration of opening mode I, II and III which is why the study of mixed-mode loads is of particular importance [7-9,14,24]. In linear fracture mechanics the principle of superposition allows to obtain solutions for mixed mode I/II crack problems whereas in nonlinear fracture mechanics many questions are still open [9-22]. Analysis of the near crack-tip fields in power-law hardening (or power-law creeping) damaged materials under mixed-mode loading results in new nonlinear eigenvalue problems in which the whole spectrum of the eigenvalues and orders of stress singularity have to be determined [20-23]. For instance, in [21] asymptotic stress, strain, and displacement distributions in the vicinity of the mixed-mode crack for the stress-state sensitive elastic materials are considered and a nontrivial solution with the eigenvalue $s=1$ in the displacement series is found explicitly. Investigation of the asymptotic behaviour of the stress, strain, and displacements in the vicinity of a mixed-mode crack in the stress-state sensitive materials leads to conclusion that the traditional approaches, such as the superposition of the solutions as well as the assumptions for the symmetrical or antisymmetrical stress distributions can not be used. Therefore, in order to obtain the crack tip fields it is necessary to solve a new eigenvalue problem which in general case can be nonlinear. Nevertheless, in this work, a nontrivial solution with $s=1$ in the displacement series is found explicitly that demonstrates such specific features as the volume change under the condition of the remote shear loading and so on. It is shown [21] that the stronger the material stress-state-sensitivity is the stronger the resulting stress, strain and displacement fields deviate from the linear elastic ones. In [22] the stress field in the closest vicinity of a sharp material inclusion tip is characterized by 1 or 2 singular exponents. The exponents are calculated as an eigenvalue problem. The stress description by only one or two terms is not sufficient and leads to misleading results.

The objective of this study is to analyze the crack-tip fields in a damaged material under mixed-mode loading conditions and to consider the meso-mechanical effect of damage on the stress-strain state near the crack tip. The method proposed has been applied to nonlinear eigenvalue problems arising from the problem of the determining the near crack-tip fields in the damaged 
materials. In continuum damage mechanics $[2,6,7,10,12,13,16]$, the damage state at an arbitrary point in the material is represented by a properly defined integrity (continuity) variable $\psi(r, \theta)$. The integrity parameter reaches its critical value at fracture. According to this notion, a crack in a fracture process can be modeled with the concept of a completely damaged zone in the vicinity of the crack tip. Namely a crack can be represented by a region where the integrity state has attained to its critical state $\psi=\psi_{c r}$, i.e., by the completely damaged zone (CDZ) [6]. Then the development of the crack and its preceding damage can be elucidated by analyzing the local states of stress, strain and damage. The CDZ may be interpreted as the zone of critical decrease in the effective area due to damage development. Inside the completely damaged zone the damage involved reaches its critical value (for instance, the damage parameter reaches unity) and a complete fracture failure occurs. In view of material damage stresses are relaxed to vanishing [6,9,24,25]. Therefore, one can assume that the stress components in the CDZ equal zero. Outside the zone damage alters the stress distribution substantially compared to the corresponding non-damaging material. Well outside the CDZ the continuity parameter is equal to 1. Asymptotic remote boundary conditions are the asymptotic approaching the HRR solution. Dimensional analysis of the system formulated shows that the damage mechanics equations must have similarity solutions $[9,24]$. The present paper extends works $[19,20,23,24,26]$ and constructs the asymptotic stress and continuity fields for stationary mixed-mode crack in damaged media under creep conditions.

\section{Mixed mode crack problem: mathematical statement of the problem and fundamental equations}

Static mixed mode crack problems under plane stress and plane strain conditions under creep regime are considered. The equilibrium equations and compatibility condition in the polar coordinate system can, respectively, be written as

$$
\begin{aligned}
& r \sigma_{r r, r}+\sigma_{r \theta, \theta}+\sigma_{r r}-\sigma_{\theta \theta}=0, \quad \sigma_{\theta \theta}+r \sigma_{r \theta, r}+2 \sigma_{r \theta}=0, \\
& 2\left(r \varepsilon_{r \theta, \theta}\right)_{, r}=\varepsilon_{r r, \theta \theta}-r \varepsilon_{r r, r}+r\left(r \varepsilon_{\theta \theta}\right)_{, r r} .
\end{aligned}
$$

The constitutive equations are described by the power law stress-strain rate relations incorporating the damage state parameter $\omega=1-\psi$ and the creep strain rate is defined as follows

$$
\varepsilon_{i j}=3\left(\sigma_{e} / \psi\right)^{n-1} s_{i j} /(2 \psi)
$$

where $s_{i j}=\sigma_{i j}-\sigma_{k k} \delta_{i j} / 3$ are the deviatoric stress tensor components; $B, n$ are material constants which control secondary creep behavior and can be determined from a log-log plot of the creep strain rate vs the applied stress; $\psi$ is an integrity (continuity) parameter ( $\psi=1$ indicates no damage and $\psi=0$ corresponds to complete damage); $\varepsilon_{i j}$ are the strain components which for the plane stress and plane strain conditions take the form:

$$
\begin{array}{lll}
\varepsilon_{r r}=B \sigma_{e}^{n-1}\left(2 \sigma_{r r}-\sigma_{\theta \theta}\right) /\left(2 \psi^{n}\right), & \varepsilon_{\theta \theta}=B \sigma_{e}^{n-1}\left(2 \sigma_{\theta \theta}-\sigma_{r r}\right) /\left(2 \psi^{n}\right), & \varepsilon_{r \theta}=3 B \sigma_{e}^{n-1} \sigma_{r \theta} /\left(2 \psi^{n}\right), \\
\varepsilon_{r r}=3 B \sigma_{e}^{n-1}\left(\sigma_{r r}-\sigma_{\theta \theta}\right) /\left(4 \psi^{n}\right), & \varepsilon_{\theta \theta}=3 B \sigma_{e}^{n-1}\left(\sigma_{\theta \theta}-\sigma_{r r}\right) /\left(4 \psi^{n}\right), & \varepsilon_{r \theta}=3 B \sigma_{e}^{n-1} \sigma_{r \theta} /\left(2 \psi^{n}\right) .
\end{array}
$$

The von-Mises equivalent stress is expressed by $\sigma_{e}=\sqrt{\sigma_{r r}^{2}+\sigma_{\theta \theta}^{2}-\sigma_{r r} \sigma_{\theta \theta}+3 \sigma_{r \theta}^{2}}$ and $\sigma_{e}=(\sqrt{3} / 2) \sqrt{\left(\sigma_{r r}-\sigma_{\theta \theta}\right)^{2}+4 \sigma_{r \theta}^{2}} \quad$ for plane stress and plane strain conditions respectively. The constitutive model (3) is the phenomenological model of Kachanov and Rabotnov widely employed in creep damage theory and in damage analysis of high temperature structures [1, 6, 18, 26, 27]. The material parameters pertinent to equations (2) for copper, the aluminium alloy, ferritic steels obtained from creep curves are given by Riedel in [18]. By noting that the creep damage is brought about by the development of microscopic voids in creep process, L.M. Kachanov represented the damage state by a scalar integrity variable $\psi(0 \leq \psi \leq 1)$ where $\psi=1$ and $\psi=0$ signify the initial undamaged state and the final completely damaged state (or final fractured state), respectively [6,12,13,26]. L.M. Kachanov [27] described the damage development by means of an evolution equation

$$
d \psi / d t=-A\left(\sigma_{e q v} / \psi\right)^{m},
$$

where $A$ and $m$ are material constants which control tertiary creep behavior, $\sigma_{e q v}=\alpha \sigma_{1}+\beta \sigma_{e}+\gamma \sigma_{k k}$, $\sigma_{1}$ is the maximum principal stress, $\sigma_{k k}$ is the hydrostatic stress; $\alpha$ is the material constant, which describes the effect of the multi-axial stress state behavior of material and ranges from $\alpha=0$ (equivalent stress dominant) to $\alpha=1$ (maximum principal stress dominant). The accurate prediction of $\alpha$ value plays an important role in the application of the multi-axial Kachanov-Rabotnov damage model. The solution of Eqs. (1) - (5) should satisfy the traditional traction free boundary conditions on the crack surfaces

$$
\sigma_{r \theta}(r, \theta= \pm \pi)=0, \quad \sigma_{\theta \theta}(r, \theta= \pm \pi)=0
$$

The mixed-mode loading can be characterized in terms of the mixity parameter $M^{p}[5,7,24]$ which is defined as

$$
M^{p}=\frac{2}{\pi} \arctan \left(\lim _{r \rightarrow 0}\left|\frac{\sigma_{\theta \theta}(r, \theta=0)}{\sigma_{r \theta}(r, \theta=0)}\right|\right) .
$$

The mixity parameter $M^{p}$ equals 0 for pure mode II; 1 for pure mode I, and $0<M^{p}<1$ for different mixities of modes I and II. Thus, for combine-mode fracture the mixity parameter $M^{p}$ completely specifies the near-crack-tip fields for a given value of the creep exponent. 


\section{Asymptotic solution}

One can assume that the completely damaged zone in the vicinity of the crack tip evolves during the deformation of the cracked body. The asymptotic solution for the stress-strain fields and the continuity field can be found outside the completely damaged zone. As the continuity parameter reaches at infinity the value corresponding to the undamaged materials it is possible to realize the approach described below.

\subsection{Similarity solution}

Dimensional analysis of the system formulated shows that the damage mechanics equations must have similarity solutions of the form [18]

$$
\sigma_{i j}(r, \theta, t)=(A t)^{-1 / m} \hat{\sigma}_{i j}(R, \theta), \quad \psi(r, \theta, t)=\hat{\psi}(R, \theta)
$$

where $R=r(A t)^{-(n+1) / m} B I_{n} / C^{*}$ is the similarity variable. It should be noted that the remote boundary conditions can be formulated in a more general form $\sigma_{i j}(r \rightarrow \infty, \theta, t)=\widetilde{C} r^{s} \bar{\sigma}_{i j}(\theta, n)$, where the stress singularity exponent $s$ is unknown and has to be determined as a part of solution, $C$ is the amplitude of the stress field at infinity defined by the specimen configuration and loading conditions. For the power-law creep constitutive relations, the power-law damage evolution equation and the more general remote boundary conditions the self-similar variable $R=r(A t)^{1 /(s m)} \widetilde{C}$ can be introduced. After introducing the selfsimilar variable the equilibrium equations, the constitutive equations, the compatibility condition retain their forms, whereas the damage evolution equation becomes

$$
R \hat{\psi}_{R}=-\operatorname{sm}\left(\hat{\sigma}_{e} / \hat{\psi}\right)^{m}
$$

(the superscript ${ }^{\wedge}$ is further omitted). By postulating the Airy stress function $\chi(R, \theta)$ expressed in the polar coordinate system, the stress components state are expressed as: $\sigma_{\theta \theta}=\chi,{ }_{R R}, \sigma_{R R}=\chi,{ }_{R} / R+\chi,{ }_{\theta \theta} / R^{2}, \sigma_{R \theta}=-\left(\chi,{ }_{\theta} / R\right),{ }_{R}$.

The asymptotic solution outside the completely damaged zone $(R \rightarrow \infty)$ is sought in the form

$$
\chi(R, \theta)=\sum_{j=0}^{\infty} R^{\lambda_{j}+1} f_{j}(\theta), \quad \psi(r, \theta)=1-\sum_{j=0}^{\infty} R^{\gamma_{j}} g_{j}(\theta) .
$$

In view of (11) the asymptotic presentation of the stress tensor components has the form

$$
\sigma_{i j}(R, \theta)=\sum_{k=0}^{\infty} R^{\lambda_{k}-1} \sigma_{i j}^{(k)}(\theta), \quad \sigma_{R R}^{(k)}(\theta)=\left(\lambda_{k}+1\right) f_{k}(\theta)+f_{k}^{\prime \prime}(\theta), \quad \sigma_{R \theta}^{(k)}(\theta)=-\left(\lambda_{k}+1\right) f_{k}^{\prime}(\theta), \quad \sigma_{\theta \theta}^{(k)}(\theta)=\lambda_{k}\left(\lambda_{k}+1\right) f_{k}(\theta) .
$$

It can be shown that the asymptotic series expansion of the strain rate tensor components can be written as:

$$
\varepsilon_{i j}(R, \theta)=\sum_{k=0}^{\infty} R^{\left(\lambda_{0}-1\right)(n+k m)} \varepsilon_{i j}^{(k)}(\theta) .
$$

\subsection{Structure of the asymptotic solution. The leading term of the asymptotic governing equations}

First consider the leading terms of the asymptotic expansions (11): $\chi(R, \theta)=r^{\lambda_{0}+1} f_{0}(\theta), \psi=1$ where $\lambda_{0}$ is indeterminate exponent and $f_{0}(\theta)$ is an indeterminate function of the polar angle, respectively. In view of the asymptotic presentation for the Airy stress potential (11) the asymptotic stress field at the crack tip is derived as follows $\sigma_{i j}(R, \theta)=R^{\lambda_{0}-1} \sigma_{i j}(\theta)$, where $\lambda_{0}-1$ denotes the exponent representing the singularity of the stress field, and will be called the stress singularity exponent hereafter. According to Eq. 13 the asymptotic strain field as $R \rightarrow \infty$ takes the form $\varepsilon_{i j}(R, \theta)=B R^{\left(\lambda_{0}-1\right) n} \varepsilon_{i j}^{(0)}(\theta)$. The compatibility condition (Eq. 2) results in the nonlinear forth-order ordinary differential equation (ODE) for the function $f_{0}(\theta)$ :

$$
\begin{aligned}
& f_{e}^{2} f_{0}^{I V}\left\{(n-1)\left[\left(1-\lambda_{0}^{2}\right) f_{0}+f_{0}^{\prime \prime}\right]^{2}+f_{e}^{2}\right\}+(n-1)(n-3)\left\{\left[\left(1-\lambda_{0}^{2}\right) f_{0}+f_{0}^{\prime \prime}\right]\left[\left(1-\lambda_{0}^{2}\right) f_{0}^{\prime}+f_{0}^{\prime \prime \prime}\right]+4 \lambda^{2} f_{0}^{\prime} f_{0}^{\prime \prime}\right\}^{2}\left[\left(1-\lambda_{0}^{2}\right) f_{0}+f_{0}^{\prime \prime}\right]+ \\
& +(n-1) f_{e}^{2}\left\{\left[\left(1-\lambda_{0}^{2}\right) f_{0}^{\prime}+f_{0}^{\prime \prime \prime}\right]^{2}+\left[\left(1-\lambda_{0}^{2}\right) f_{0}+f_{0}^{\prime \prime}\right]\left(1-\lambda_{0}^{2}\right) f_{0}^{\prime \prime}+4 \lambda_{0}^{2}\left(f_{0}^{\prime \prime 2}+f_{0}^{\prime} f_{0}^{\prime \prime \prime}\right)\right\}\left[\left(1-\lambda_{0}^{2}\right) f_{0}+f_{0}^{\prime \prime}\right]+ \\
& +2(n-1) f_{e}^{2}\left\{\left[\left(1-\lambda_{0}^{2}\right) f_{0}+f_{0}^{\prime \prime}\right]\left[\left(1-\lambda_{0}^{2}\right) f_{0}^{\prime}+f_{0}^{\prime \prime \prime}\right]+4 \lambda_{0}^{2} f_{0}^{\prime} f_{0}^{\prime \prime}\right\}\left[\left(1-\lambda_{0}^{2}\right) f_{0}^{\prime}+f_{0}^{\prime \prime \prime}\right]+ \\
& +\tilde{N}_{1}(n-1) f_{e}^{2}\left\{\left[\left(1-\lambda_{0}^{2}\right) f_{0}+f_{0}^{\prime \prime}\right]\left[\left(1-\lambda_{0}^{2}\right) f_{0}^{\prime}+f_{0}^{\prime \prime \prime}\right]+4 \lambda_{0}^{2} f_{0}^{\prime} f_{0}^{\prime \prime}\right\} f_{0}^{\prime}+ \\
& +C_{1} f_{e}^{4} f_{0}^{\prime \prime}-C_{2} f_{e}^{4}\left[\left(1-\lambda_{0}^{2}\right) f_{0}+f_{0}^{\prime \prime}\right]+f_{e}^{4}\left(1-\lambda_{0}^{2}\right) f_{0}^{\prime \prime}=0
\end{aligned}
$$

where the following notations are adopted $f_{e}^{2}=\left[\left(1-\lambda_{0}^{2}\right) f_{0}+f_{0}^{\prime \prime}\right]^{2}+4 \lambda_{0}^{2} f_{0}^{2}, C_{1}=4 \lambda_{0}\left[\left(\lambda_{0}-1\right) n+1\right], C_{2}=\left(\lambda_{0}-1\right) n\left[\left(\lambda_{0}-1\right) n+1\right]$ for plane strain conditions; 


$$
\begin{aligned}
& f^{I V} f_{e}^{2}\left\{(n-1)\left[\left(\lambda_{0}+1\right)\left(2-\lambda_{0}\right) f_{0}+2 f_{0}^{\prime \prime}\right]^{2} / 2+2 f_{e}^{2}\right\}+6\left[\left(\lambda_{0}-1\right) n+1\right] \lambda_{0}\left\{(n-1) f_{e}^{2} h f^{\prime}+f_{e}^{4} f^{\prime \prime}\right\}+(n-1)(n-3) h^{2} \times \\
& \times\left[\left(\lambda_{0}+1\right)\left(2-\lambda_{0}\right) f_{0}+2 f_{0}^{\prime \prime}\right]+(n-1) f_{e}^{2}\left[\left(\lambda_{0}+1\right)\left(\lambda_{0}+2\right) f_{0}+2 f_{0}^{\prime \prime}\right]\left\{\left[\left(\lambda_{0}+1\right) f_{0}^{\prime}+f_{0}^{\prime \prime \prime}\right]^{2}+\left[(\lambda+1) f+f^{\prime \prime}\right](\lambda+1) f^{\prime \prime}+\right. \\
& +\left(\lambda_{0}+1\right)^{2} \lambda_{0}^{2}\left(f_{0}^{\prime 2}+f f^{\prime \prime}\right)-\left(\lambda_{0}+1\right)^{2} \lambda_{0} f_{0} f_{0}^{\prime \prime} / 2-\left[\left(\lambda_{0}+1\right) f_{0}^{\prime}+f_{0}^{\prime \prime \prime}\right]\left(\lambda_{0}+1\right) \lambda_{0} f_{0}^{\prime}+f_{e}^{4}\left(\lambda_{0}+1\right)\left(2-\lambda_{0}\right) f_{0}^{\prime \prime}- \\
& \left.-\left[(\lambda+1) f+f^{\prime \prime}\right](\lambda+1) \lambda f^{\prime \prime}+3 \lambda_{0}^{2}\left(f_{0}^{\prime \prime 2}+f_{0}^{\prime} f_{0}^{\prime \prime \prime}\right)\right\}+2(n-1) f_{e}^{2} h\left[\left(\lambda_{0}+1\right)\left(2-\lambda_{0}\right) f_{0}^{\prime}+2 f_{0}^{\prime \prime \prime}\right] / 2- \\
& -(\lambda-1) n f_{e}^{4}\left[(\lambda+1)(2-\lambda) f_{0}+2 f_{0}^{\prime \prime}\right]+\left[\left(\lambda_{0}-1\right) n+1\right]\left(\lambda_{0}-1\right) n f_{e}^{4}\left[\left(\lambda_{0}+1\right)\left(2 \lambda_{0}-1\right) f_{0}-f_{0}^{\prime \prime}\right]=0,
\end{aligned}
$$

where the following notations are adopted

$$
\begin{aligned}
& f_{e}=\sqrt{\left[\left(\lambda_{0}+1\right) f_{0}+f_{0}^{\prime \prime}\right]^{2}+\left(\lambda_{0}+1\right)^{2} \lambda_{0}^{2} f_{0}^{2}-\left[\left(\lambda_{0}+1\right) f_{0}+f_{0}^{\prime \prime}\right]\left(\lambda_{0}+1\right) \lambda_{0} f_{0}+3 \lambda_{0}^{2}\left(f_{0}^{\prime}\right)^{2}}, \quad h=\left[\left(\lambda_{0}+1\right) f_{0}+f_{0}^{\prime \prime}\right] \times \\
& \times\left[\left(\lambda_{0}+1\right) f_{0}^{\prime}+f_{0}^{\prime \prime \prime}\right]+\left(\lambda_{0}+1\right)^{2} \lambda_{0}^{2} f_{0} f_{0}^{\prime}-\left[\left(\lambda_{0}+1\right) f_{0}^{\prime}+f_{0}^{\prime \prime \prime}\right]\left(\lambda_{0}+1\right) \lambda_{0} f_{0} / 2-\left[\left(\lambda_{0}+1\right) f_{0}+f_{0}^{\prime \prime}\right]\left(\lambda_{0}+1\right) \lambda_{0} f_{0}^{\prime} / 2+3 \lambda_{0}^{2} f_{0}^{\prime} f_{0}^{\prime \prime}
\end{aligned}
$$

for plane stress conditions respectively. The boundary conditions follow from the traction-free boundary conditions:

$$
f_{0}(\theta= \pm \pi)=0, \quad f_{0}^{\prime}(\theta= \pm \pi)=0 .
$$

Hence the governing equations (1) - (6) are transformed into nonlinear eigenvalue problems of ordinary differential equations (ODE) with respect to the circumferential coordinate around the crack tip. Thus it is necessary to find the nontrivial solutions of (14) and (15) satisfying the boundary conditions (16).

\section{Nonlinear eigenvalue problems. Numerical results. Eigenspectra of nonlinear eigenvalue problems}

The method is further developed in the present work to analyze nonlinear eigenvalue problems of ODE for equations (14) and (15). The stress singularity orders $\lambda_{0}$ and the associated eigenfunctions $f_{0}(\theta)$ for plane strain and plane stress conditions are obtained. In general, most of the existing methods have their own merits and are complementary, depending on the nature of the problems to be solved. To solve the two-point boundary value problems an efficient method to deal with eigenvalue problems of

\begin{tabular}{|c|c|c|c|c|c|}
\hline Mixity parameter & $\lambda_{0}$ & $f_{0}^{\prime \prime}(0)$ & $f_{0}^{\prime \prime \prime}(0)$ & $f_{0}^{\prime \prime}(-\pi)$ & $f_{0}^{\prime \prime \prime}(-\pi)$ \\
\hline 0.95 & -0.261580 & 0.165263 & -0.783132 & 0.911946 & -0.694200 \\
\hline 0.9 & -0.260520 & 0.160476 & -0.817484 & 0.813416 & -0.617651 \\
\hline 0.8 & -0.256052 & 0.144177 & -0.838728 & 0.589120 & -0.442762 \\
\hline 0.7 & -0.248870 & 0.126642 & -0.831073 & 0.372380 & -0.272113 \\
\hline 0.6 & -0.240700 & 0.109252 & -0.814365 & 0.161493 & -0.090100 \\
\hline 0.5 & -0.230512 & 0.082163 & -0.787752 & -0.101438 & 0.113079 \\
\hline 0.4 & -0.226700 & 0.083062 & -0.782363 & -0.155373 & 0.125205 \\
\hline 0.3 & -0.227669 & 0.095646 & -0.801477 & -0.213141 & 0.161948 \\
\hline 0.2 & -0.235040 & 0.104379 & -0.822132 & -0.280236 & 0.210731 \\
\hline 0.1 & -0.250783 & 0.093861 & -0.837159 & -0.369869 & 0.280496 \\
\hline 0.05 & -0.262025 & 0.067491 & -0.805601 & -0.431242 & 0.329927 \\
\hline
\end{tabular}
ODE is apparently needed. In this paper the eigenvalues of the nonlinear eigenvalue problems are obtained by the technique developed in [24]. The algorithm is intended for mixed mode loading when the eigenvalue is not known and should be determined as a part of the solution. The mail idea is to find eigenvalues different from the classical eigenvalue corresponding to the HRR problem $\lambda_{0}=n /(n+1)$. To find new eigenvalues an additional requirement following from physical or mathematical considerations is need. The new condition of continuity of the radial stress on the line extending the crack is used. The numerical results are given in Tables 1-4 for plane strain conditions and in Tables 5-8 for plane stress conditions respectively.

\begin{tabular}{llllll}
\multicolumn{6}{c}{ Table 1. Eigenvalues of the nonlinear eigenvalue problem for $n=2$ (plane strain conditions) } \\
\hline Mixity parameter & $\lambda_{0}$ & $f_{0}^{\prime \prime}(0)$ & $f_{0}^{\prime \prime \prime}(0)$ & $f_{0}^{\prime \prime}(-\pi)$ & $f_{0}^{\prime \prime}(-\pi)$ \\
\hline 0.95 & -0.309869 & -0.006883 & -0.409577 & 0.746037 & -0.292556 \\
0.9 & -0.309310 & -0.007461 & -0.344635 & 0.777310 & -0.313217 \\
0.8 & -0.306884 & -0.009687 & -0.210585 & 0.786405 & -0.327997 \\
0.7 & -0.302045 & -0.015423 & -0.089789 & 0.757415 & -0.321367 \\
0.6 & -0.290875 & -0.033625 & 0.019798 & 0.702499 & -0.298084 \\
0.5 & -0.282566 & -0.036275 & 0.108960 & 0.650499 & -0.275722 \\
0.4 & -0.274875 & -0.024113 & 0.186358 & 0.590347 & -0.249031 \\
0.3 & -0.271672 & -0.007071 & 0.260397 & 0.543846 & -0.230473 \\
0.2 & -0.272741 & 0.007771 & 0.337598 & 0.500515 & -0.215603 \\
0.1 & -0.275936 & 0.012592 & 0.442771 & 0.459684 & -0.203360 \\
0.05 & -0.277383 & 0.008378 & 0.518376 & 0.437743 & -0.196991 \\
\hline
\end{tabular}


Mathematical Modeling / L. Stepanova, E. Mironova

Table 3. Eigenvalues of the nonlinear eigenvalue problem for $n=4$ (plane strain conditions).

\begin{tabular}{llllll}
\hline Mixity parameter & $\lambda_{0}$ & $f_{0}^{\prime \prime}(0)$ & $f_{0}^{\prime \prime \prime}(0)$ & $f_{0}^{\prime \prime}(-\pi)$ & $f_{0}^{\prime \prime \prime}(-\pi)$ \\
\hline 0.95 & -0.235100 & 0.236297 & -0.928023 & 1.014212 & -0.898791 \\
0.9 & -0.234510 & 0.234943 & -0.965785 & 0.912209 & -0.808333 \\
0.8 & -0.227530 & 0.212686 & -0.974785 & 0.667675 & -0.587889 \\
0.7 & -0.220460 & 0.191738 & -0.955971 & 0.440489 & -0.384975 \\
0.6 & -0.214380 & 0.171747 & -0.928916 & 0.232746 & -0.200054 \\
0.5 & -0.206813 & 0.139765 & -0.888099 & -0.072201 & 0.0926523 \\
0.4 & -0.204496 & 0.132479 & -0.867790 & -0.132744 & 0.116858 \\
0.3 & -0.206710 & 0.142094 & -0.879023 & -0.198359 & 0.172059 \\
0.2 & -0.215000 & 0.149162 & -0.898657 & -0.275789 & 0.240456 \\
0.1 & -0.233547 & 0.133510 & -0.937100 & -0.380728 & 0.337187 \\
0.05 & -0.249140 & 0.101767 & -0.947125 & -0.457725 & 0.410773 \\
\hline
\end{tabular}

Table 4. Eigenvalues of the nonlinear eigenvalue problem for $n=5$ (plane strain conditions)

\begin{tabular}{|c|c|c|c|c|c|}
\hline Mixity parameter & $\lambda_{0}$ & $f_{0}^{\prime \prime}(0)$ & $f_{0}^{\prime \prime \prime}(0)$ & $f_{0}^{\prime \prime}(-\pi)$ & $f_{0}^{\prime \prime \prime}(-\pi)$ \\
\hline 0.95 & -0.221500 & 0.285827 & -1.027835 & 1.074981 & -1.024605 \\
\hline 0.9 & -0.218248 & 0.276260 & -1.055626 & 0.958976 & -0.911524 \\
\hline 0.8 & -0.210625 & 0.252063 & -1.058479 & 0.706036 & -0.6667539 \\
\hline 0.7 & -0.204759 & 0.230065 & -1.034373 & 0.474972 & -0.446257 \\
\hline 0.6 & -0.200055 & 0.208164 & -1.00100 & 0.266302 & -0.248949 \\
\hline 0.5 & -0.194290 & 0.175457 & -0.954079 & -0.053984 & 0.0932645 \\
\hline 0.4 & -0.192320 & 0.160817 & -0.921897 & -0.120061 & 0.112156 \\
\hline 0.3 & -0.194812 & 0.168460 & -0.927342 & -0.190532 & 0.177562 \\
\hline 0.2 & -0.202342 & 0.173107 & -0.940975 & -0.274124 & 0.257060 \\
\hline 0.1 & -0.221158 & 0.155956 & -0.989844 & -0.387606 & 0.369339 \\
\hline 0.05 & -0.237872 & 0.121203 & -1.040272 & -0.473791 & 0.457832 \\
\hline
\end{tabular}

\begin{tabular}{llllll}
\multicolumn{6}{c}{ Table 5. Eigenvalues of the nonlinear eigenvalue problem for $n=2$ (plane stress conditi } \\
\hline Mixity parameter & $\lambda_{0}$ & $f_{0}^{\prime \prime}(0)$ & $f_{0}^{\prime \prime \prime}(0)$ & $f_{0}^{\prime \prime}(-\pi)$ & $f_{0}^{\prime \prime}(-\pi)$ \\
\hline 0.95 & -0.302240 & -0.178386 & -0.327269 & 0.3355941 & 0.227941 \\
0.9 & -0.300320 & -0.178070 & -0.366381 & 0.2575700 & 0.292668 \\
0.8 & -0.286090 & -0.192771 & -0.407730 & -0.088471 & 0.769239 \\
0.7 & -0.267890 & -0.215107 & -0.429407 & -0.202419 & 0.293261 \\
0.6 & -0.260930 & -0.209033 & -0.466896 & -0.244312 & 0.207239 \\
0.5 & -0252332 & -0.193741 & -0.519713 & -0.270032 & 0.157534 \\
0.4 & -0.243698 & -0.168133 & -0.584743 & -0.285889 & 0.120618 \\
0.3 & -0.237019 & -0.136613 & -0.661107 & -0.302160 & 0.092347 \\
0.2 & -0.232479 & -0.096929 & -0.721807 & -0.315939 & 0.067278 \\
0.1 & -0.229872 & -0.051085 & -0.763118 & -0.331096 & 0.044413 \\
0.05 & -0.229234 & -0.026058 & -0.773639 & -0.338664 & 0.033298 \\
\hline
\end{tabular}

Table 6. Eigenvalues of the nonlinear eigenvalue problem for $n=4$ (plane stress conditions).

\begin{tabular}{llllll}
\hline Mixity parameter & $\lambda_{0}$ & $f_{0}^{\prime \prime}(0)$ & $f_{0}^{\prime \prime \prime}(0)$ & $f_{0}^{\prime \prime}(-\pi)$ & $f_{0}^{\prime \prime \prime}(-\pi)$ \\
\hline 0.95 & -0.25900 & 0.046297 & -0.554192 & 0.728468 & -0.356792 \\
0.9 & -0.25560 & 0.044068 & -0.588519 & 0.643051 & -0.313277 \\
0.8 & -0.24450 & 0.038444 & -0.620481 & 0.448631 & -0.213325 \\
0.7 & -0.23350 & 0.033607 & -0.630460 & 0.251112 & -0.103504 \\
0.6 & -0.22020 & 0.012148 & -0.623434 & -0.120807 & 0.152059 \\
0.5 & -0.21079 & 0.009305 & -0.626547 & -0.170003 & 0.103454 \\
0.4 & -0.20527 & 0.023364 & -0.636587 & -0.207179 & 0.106370 \\
0.3 & -0.20303 & 0.043891 & -0.663582 & -0.248072 & 0.119894 \\
0.2 & -0.20573 & 0.063168 & -0.697544 & -0.292794 & 0.138533 \\
0.1 & -0.21570 & 0.069677 & -0.750661 & -0.349948 & 0.165542 \\
0.05 & -0.22414 & 0.056261 & -0.777450 & -0.387722 & 0.184522 \\
\hline
\end{tabular}


Mathematical Modeling / L. Stepanova, E. Mironova

\begin{tabular}{llllll}
\multicolumn{6}{c}{ Table 7. Eigenvalues of the nonlinear eigenvalue problem for $n=6$ (plane stress condit } \\
\hline Mixity parameter & $\lambda_{0}$ & $f_{0}^{\prime \prime}(0)$ & $f_{0}^{\prime \prime}(0)$ & $f_{0}^{\prime \prime}(-\pi)$ & $f_{0}^{\prime \prime}(-\pi)$ \\
\hline 0.95 & -0.23620 & 0.092284 & -0.640557 & 0.802232 & -0.504505 \\
0.9 & -0.23000 & 0.088445 & -0.672059 & 0.707014 & -0.442238 \\
0.8 & -0.21800 & 0.084838 & -0.700964 & 0.505557 & -0.312884 \\
0.7 & -0.20880 & 0.082946 & -0.707758 & 0.314326 & -0.192580 \\
0.6 & -0.19930 & 0.069616 & -0.698358 & -0.075083 & 0.311685 \\
0.5 & -0.19078 & 0.059067 & -0.688624 & -0.137724 & 0.088869 \\
0.4 & -0.18870 & 0.071683 & -0.691122 & -0.179959 & 0.109436 \\
0.3 & -0.19332 & 0.091028 & -0.713498 & -0.227876 & 0.138206 \\
0.2 & -0.20619 & 0.106166 & -0.748785 & -0.282897 & 0.173351 \\
0.1 & -0.23041 & 0.104842 & -0.833112 & -0.356879 & 0.223327 \\
0.05 & -0.24985 & 0.084782 & -0.912969 & -0.410296 & 0.261073 \\
\hline
\end{tabular}

\begin{tabular}{llllll}
\multicolumn{6}{c}{ Table 8. Eigenvalues of the nonlinear eigenvalue problem for $n=8$ (plane stress conditions } \\
\hline Mixity parameter & $\lambda_{0}$ & $f_{0}^{\prime \prime}(0)$ & $f_{0}^{\prime \prime \prime}(0)$ & $f_{0}^{\prime \prime}(-\pi)$ & $f_{0}^{\prime \prime}(-\pi)$ \\
\hline 0.95 & -0.224000 & 0.113333 & -0.684626 & 0.831308 & -0.572658 \\
0.9 & -0.216700 & 0.109593 & -0.715353 & 0.732220 & -0.501304 \\
0.8 & -0.205400 & 0.107456 & -0.743801 & 0.528789 & -0.358568 \\
0.7 & -0.197400 & 0.107175 & -0.749584 & 0.337838 & -0.227508 \\
0.6 & -0.189810 & 0.098453 & -0.740423 & 0.126048 & -0.079175 \\
0.5 & -0.181760 & 0.082367 & -0.723195 & -0.123024 & 0.083357 \\
0.4 & -0.182300 & 0.093150 & -0.723228 & -0.167982 & 0.111747 \\
0.3 & -0.191700 & 0.110578 & -0.746282 & -0.219157 & 0.146879 \\
0.2 & -0.209870 & 0.122860 & -0.785793 & -0.278818 & 0.189787 \\
0.1 & -0.238320 & 0.118773 & -0.892595 & -0.359530 & 0.250648 \\
0.05 & -0.261703 & 0.97069 & -1.035152 & -0.419102 & 0.297851 \\
\hline
\end{tabular}

\section{Results and Discussion. Shape of the completely damaged zone in the vicinity of the crack tip}

Having obtained the angular distributions of the stress, strain and continuity fields one can determine the geometry of the completely damaged zone modeled in the vicinity of the crack tip and given by multi-parameter expansion for the continuity:

$$
\begin{aligned}
& \psi(R, \theta)=1-R^{\gamma_{0}} g_{0}(\theta)=0, \\
& \psi(R, \theta)=1-R^{\gamma_{0}} g_{0}(\theta)-R^{\gamma_{1}} g_{1}(\theta)=0, \\
& \psi(R, \theta)=1-R^{\gamma_{0}} g_{0}(\theta)-R^{\gamma_{1}} g_{1}(\theta)-R^{\gamma_{2}} g_{2}(\theta)=0, \\
& \psi(R, \theta)=1-R^{\gamma_{0}} g_{0}(\theta)-R^{\gamma_{1}} g_{1}(\theta)-R^{\gamma_{2}} g_{2}(\theta)-R^{\gamma_{3}} g_{3}(\theta)=0, \\
& \psi(R, \theta)=1-R^{\gamma_{0}} g_{0}(\theta)-R^{\gamma_{1}} g_{1}(\theta)-R^{\gamma_{2}} g_{2}(\theta)-R^{\gamma_{3}} g_{3}(\theta)-R^{\gamma_{4}} g_{4}(\theta)=0, \\
& \psi(R, \theta)=1-R^{\gamma_{0}} g_{0}(\theta)-R^{\gamma_{1}} g_{1}(\theta)-R^{\gamma_{2}} g_{2}(\theta)-R^{\gamma_{3}} g_{3}(\theta)-R^{\gamma_{4}} g_{4}(\theta)-R^{\gamma_{5}} g_{5}(\theta)=0 .
\end{aligned}
$$

One can compare the boundaries of the $\mathrm{CDZ}$ given by the two-term, three-term, four-term, five-term and six-term asymptotic expansions of the integrity (continuity) parameter (Eq. 17). It is turned out that if the asymptotic remote boundary condition is postulated as the condition of the asymptotic approaching the HRR-field then the shapes of the CDZ given by the two-term asymptotic expansion and three-term asymptotic expansions differ essentially from each other. The new stress asymptotic behavior results in the contours of the CDZ which converge to the limit contour shown in Figs. 1-7. The new far field stress asymptotic can be interpreted as the intermediate stress asymptotics valid for times and distances at which effects of the initial and boundary conditions on the stress and damage distributions are lost. The geometry of the completely damage zone for different values of the mixity parameter is shown in Figs. 1-5 where $k=1,2,3,4,5$ is designed the boundary of the CDZ built by the use of the $k+1$ - term asymptotic expansion of continuity. The red line shows the boundary of the CDZ obtained by the twoterm asymptotic expansion of the integrity parameter whereas the blue line shows the boundary of the CDZ obtained by the three-term asymptotic expansion of the integrity parameter. The green line shows the boundary of the CDZ obtained by the fourterm asymptotic expansion of the integrity parameter. From Figs. 1-7 it can be seen that the boundary of the CDZ determined by the use of the $k+1$-term asymptotic expansion of continuity is very close to the boundary built by the $k$-term asymptotic expansion of the continuity parameter whereas the HRR stress field results in the boundary of the CDZ given by the two-term expansion which differs substantially from the boundary of the CDZ given by the three-term asymptotic expansion of the integrity parameter by the form and dimensions. 


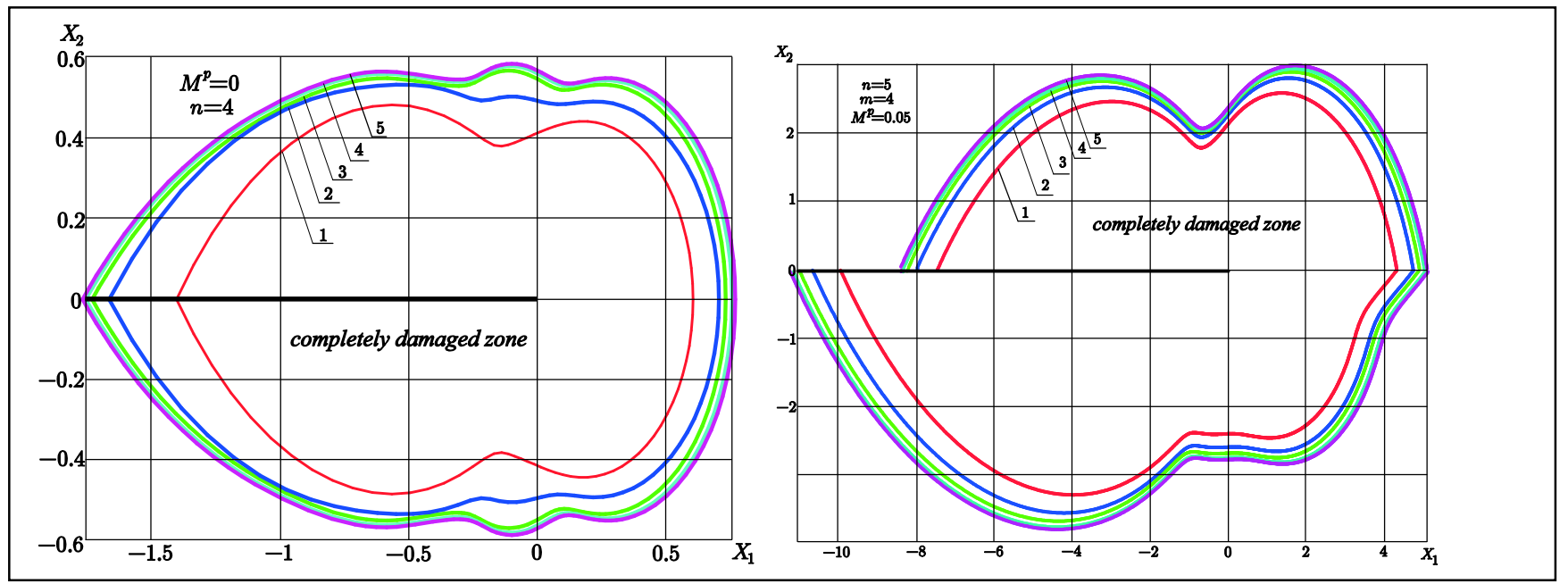

Fig.1. Geometry of the completely damaged zone in the vicinity of the crack tip for $M^{p}=0$ and $M^{p}=0.05$.
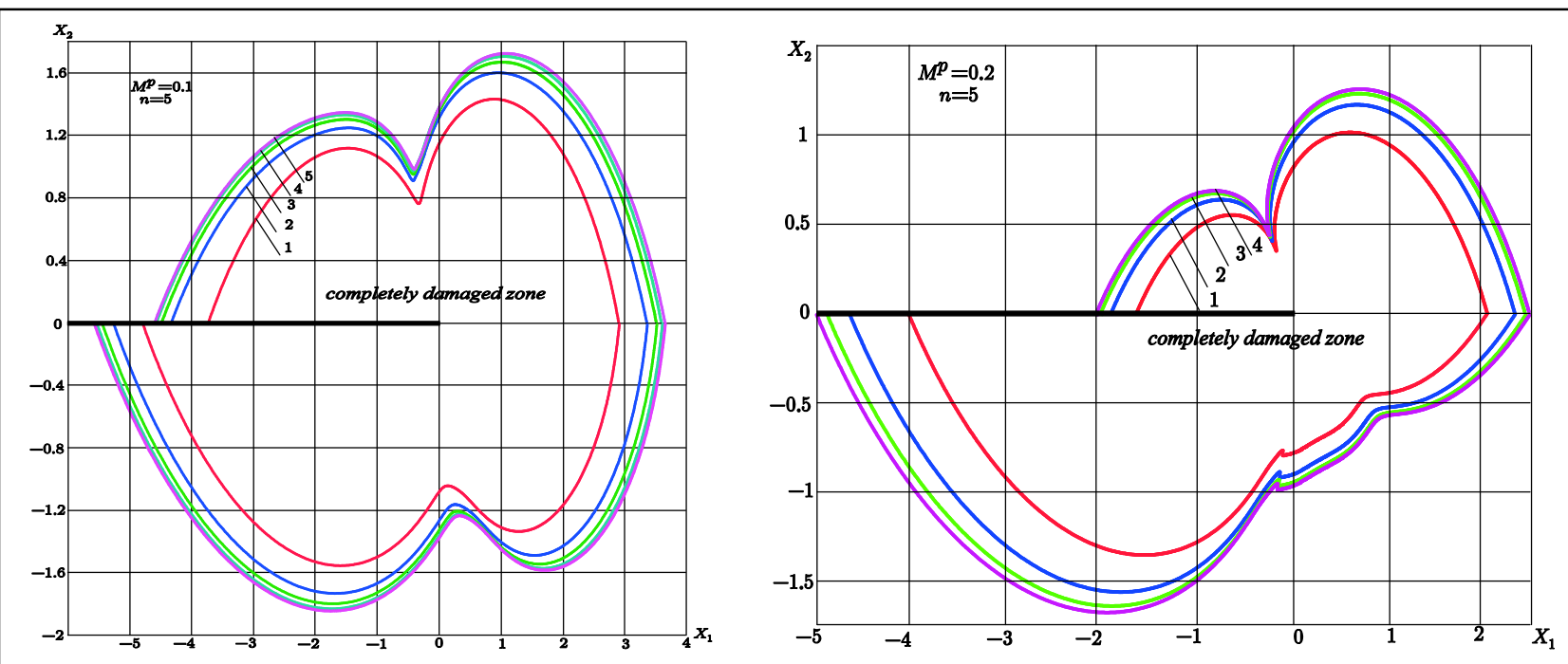

Fig. 2. The geometry of the totally damaged zone near the crack tip under mixed mode loading for $M^{p}=0.1$ and $M^{p}=0.2$.

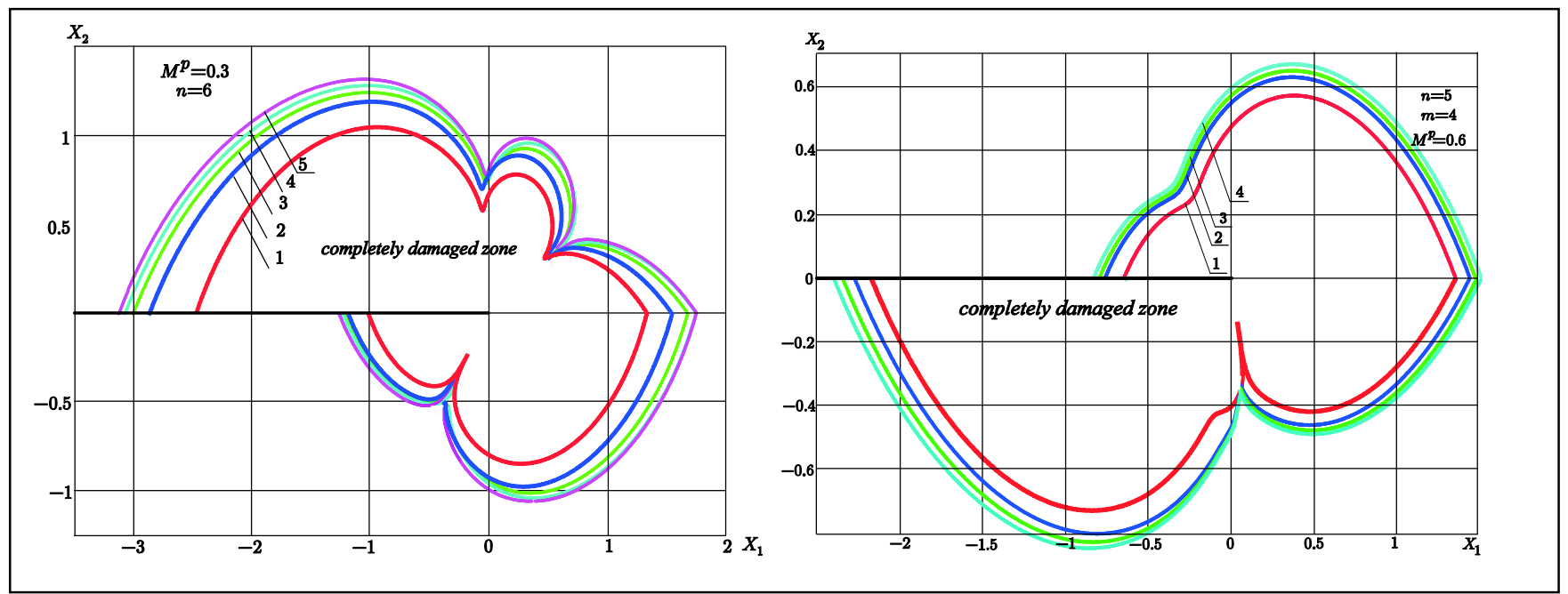

Fig.3. Geometry of the completely damaged zone in the vicinity of the crack tip under mixed mode loading for $M^{p}=0$ and $M^{p}=0.05$ (plane strain conditions). 

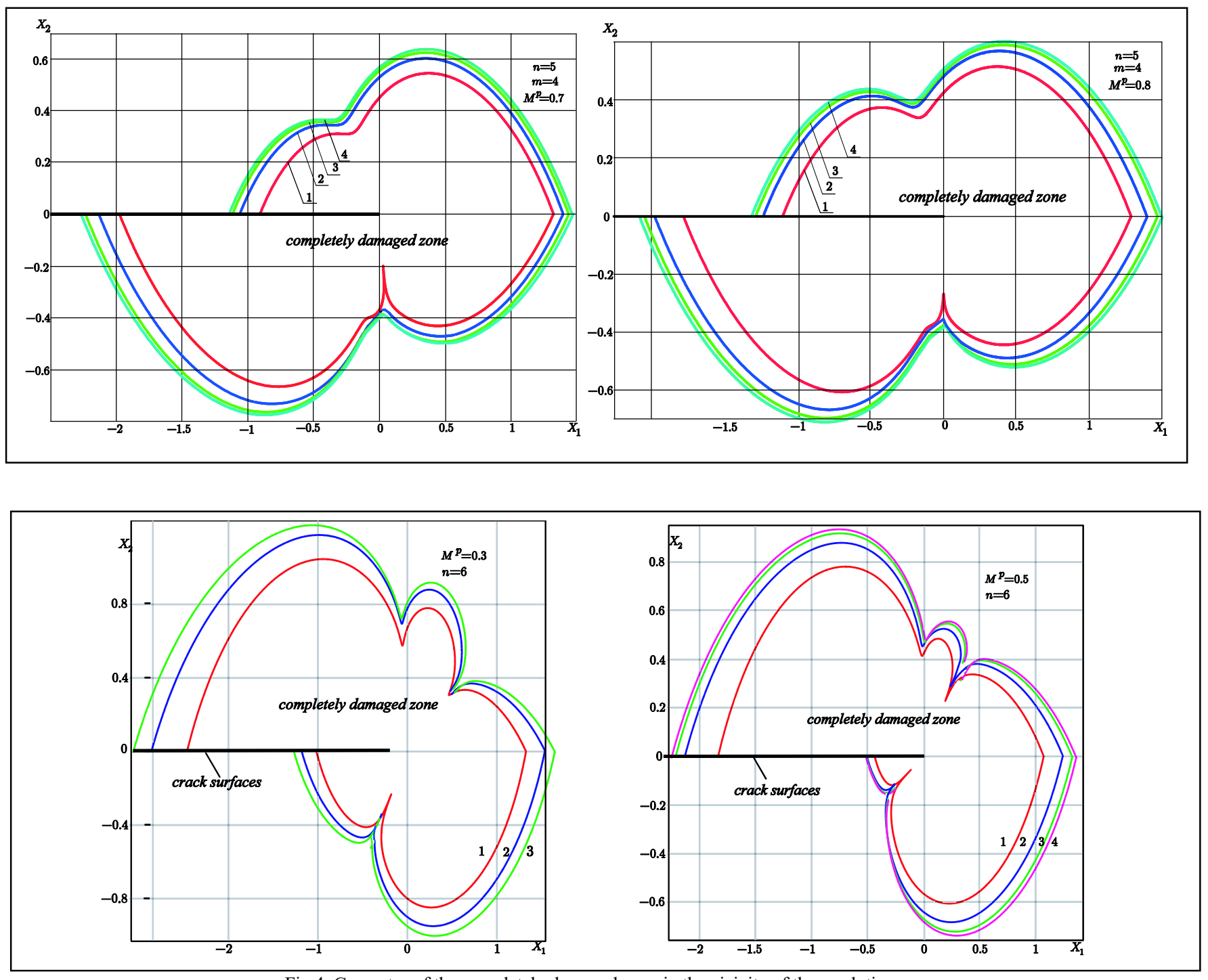

Fig.4. Geometry of the completely damaged zone in the vicinity of the crack tip.

Fig. 5. Geometry of the completely damaged zone in the vicinity of the crack tip under mixed mode loading for or $M^{p}=0.9$ and $M^{p}=1$ (plane strain conditions).

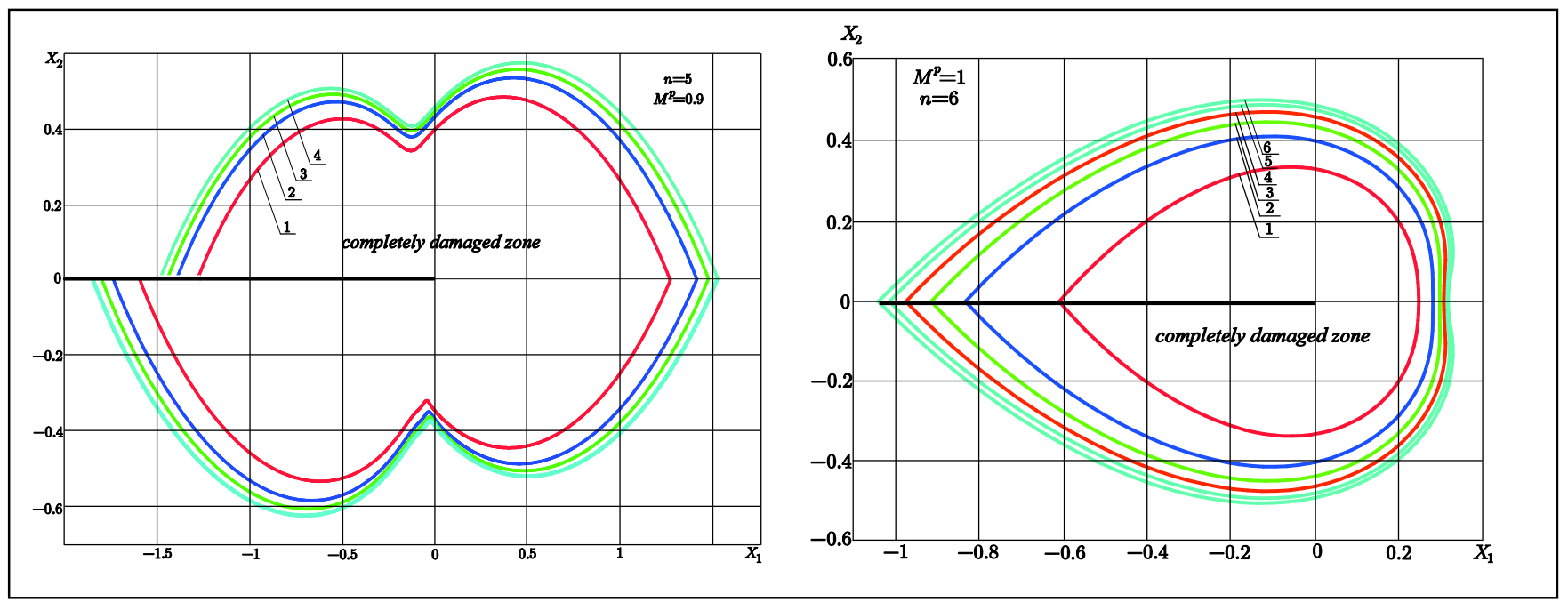

Fig. 6. Geometry of the completely damaged zone in the vicinity of the crack tip under mixed mode loading for $M^{p}=0.3$ and $M^{p}=0.5$.

\section{Conclusion}

Asymptotic crack-tip fields in damaged materials are developed for a stationary plane stress and plane strain crack under mixed mode loading conditions in a full range of the mixity parameter varying from the value corresponding to pure Mode I loading to pure Mode II loading. The asymptotic solutions are obtained by the use of the similarity variable and the similarity 
presentation of the solution. On the basis of the self-similar representation of the solution the near crack-tip stress, creep strain rate and continuity distributions are given. It is shown that meso-mechanical effect of damage accumulation near the crack tip results in new intermediate stress field asymptotic behavior and requires the solution of nonlinear eigenvalue problems. To attain eigensolutions a numerical scheme is worked out and the results obtained provide the additional eigenvalues of the HRR problem. By the use of the method proposed the whole set of eigenvalues for the mode crack in a power law material under mixed mode loading can be determined. The self-similar solutions are based on the idea of the existence of the completely damaged zone near the crack tip. The stress and creep strain rate angular functions are constructed. The higher order terms of the asymptotic expansions of stresses, creep strain rates and continuity parameter allowing to obtain the contours of the completely damaged zone in the vicinity of the crack tip are derived and investigated. The extent of the area in the vicinity of the crack tip where the material undergoes damage for the specimen under tensile loading is studied in [29 33]. The results obtained in the

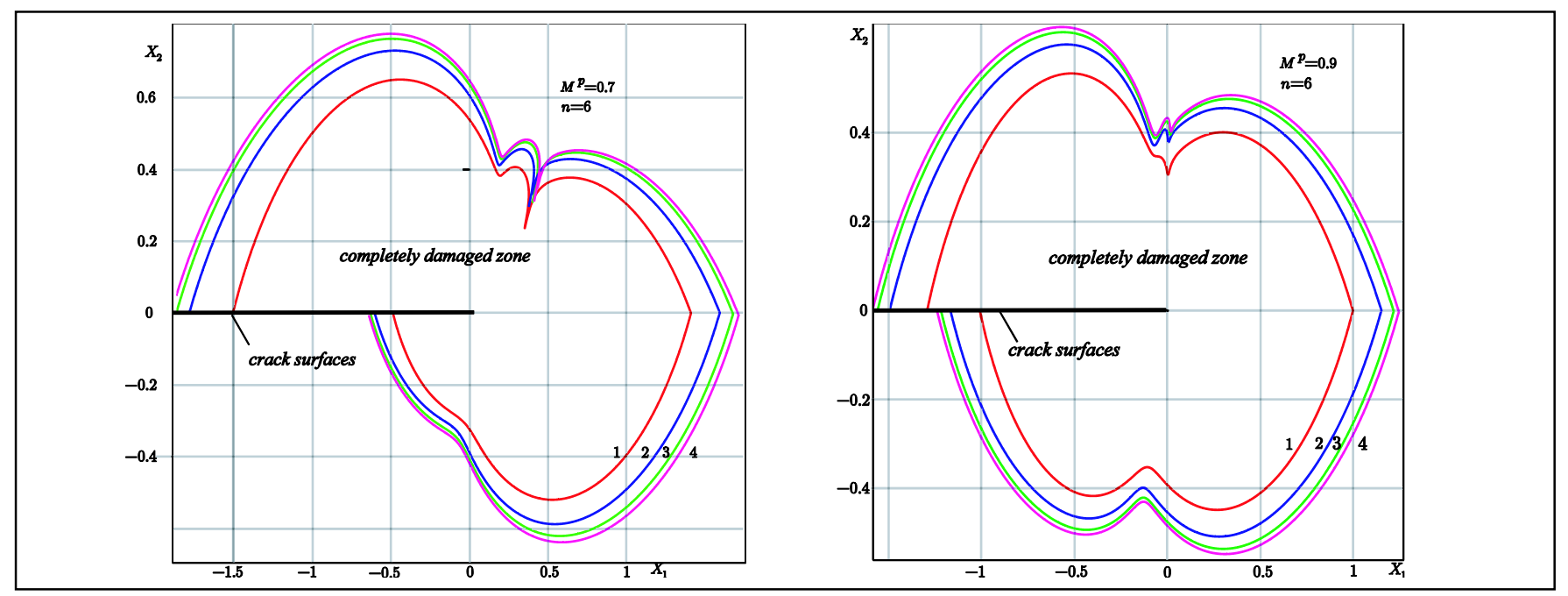

present paper are in good agreement with the results of [29-33].

Fig. 7. Geometry of the completely damaged zone near the crack tip under mixed mode loading for $M^{p}=0.7$ and $M^{p}=0.9$.

\section{Acknowledgements}

Financial support from the Russian Foundation of Basic Research (project No. 16-08-00571) is gratefully acknowledged.

\section{References}

[1] Meng Q, Weng Z. Creep damage models and their applications for crack growth analysis in pipes: A review. Engineering Fracture Mechanics 2016 : 1-30.

[2] Altenbach H, Sadowski T. Failure and Damage Analyses of Advanced Materials. Berlin: Springer, 2015.

[3] Barenblatt GI. Deformation and Fracture: Lectures on Fluid Mechanics and the Mechanics of Deformable Solids for Mathematicians and Physicists. Cambridge University Press, 2014.

[4] Bui HD. Fracture Mechanics. Inverse Problems and Solutions. Dordrecht: Springer, 2006.

[5] Stepanova LV, Roslyakov PS. Multi-parameter description of the crack-tip stress field: Analytic determination of coefficients of crack-tip stress expansions in the vicinity of the crack tips of two finite cracks in an infinite plane medium. International Journal of Solids and Structures 2016; 100-101: 10-28.

[6] Murakami S. Continuum Damage Mechanics. A Continuum Mechanics Approach to the Analysis of Damage and Fracture. Dordrecht: Springer, 2012.

[7] Kuna M. Finite Elements in Fracture Mechanics. Theory-Numerics-Applications. Dordrecht: Springer, 2013.

[8] Soyarslan C, Richter H, Bargmann S. Variants of Lemaitre damage model and their use in formability prediction of metallic materials. Mechanics of Materials 2016; 92: 58-79.

[9] Stepanova LV, Adylina EM. Stress-strain state in the vicinity of a crack under mixed loading. Journal of Applied Mechanics and Technical Physics 2014; 55(5): 885-895.

[10] Ochsner A. Continuum Damage and Fracture Mechanics. Springer Science + Business Media, Singapore, 2016.

[11] Richard HA, Schramm B, Schrimeisen N-H. Cracks on Mixed Mode loading - Theories, experiments, simulations. International Journal of Fatigue 2014; 62: $93-103$

[12] Voyiadis GZ, Kattan PI. Damage Mechanics with Finite Elements: Practical Applications with Computer Tools. Berlin: Springer, 2012.

[13] Voyiadis GZ. Handbook of Damage Mechanics. New York: Springer - Verlag, 2015.

[14] Tumanov AV, Shlyannikov VN, Kishen CJM. An automatic algorithm for mixed mode crack growth rate based on drop potential method. International Journal of Fatigue 2015; 81: 227-237.

[15] Wei RP. Fracture Mechanics. Integration of Mechanics, Materials Science and Chemistry, Cambridge University Press, 2014.

[16] Zhang W, Cai Y. Continuum Damage Mechanics and Numerical Applications. Heidelberg: Springer Science \& Business Media, 2010.

[17] Chousal JAG. M.F.S.F. de Moura, Mixed mode I+II continuum damage model applied to fracture characterization of bonded joints. Int. J. of Adhesion and Adhesives $201 ; 41: 92-97$.

[18] Riedel H. Fracture at High Temperature. Berlin: Springer-Verlag, 1987.

[19] Stepanova LV. Eigenvalue of the antiplane-shear crack problem for a power-law material. Journal of Applied Mechanics and Technical Physics 2008; 49(1): 142-147.

[20] Stepanova LV. Eigenspectra and orders of stress singularity at a mode I crack tip for a power-law medium. Comptes Rendus. Mecanique 2008; 336(1-2): 232-237.

[21] Beliakova TA, Kulagin VA. The eigenspectrum approach and T-stress at the mixed-mode crack tip for a stress-state-dependent material. Procedia Materials Science 2014; 3: 147-152.

[22] Krepl O, Klusak J. Reconstruction of a 2D stress field around the tip of a sharp material inclusion. Procedia Structural Integrity 2016; 2: $1920-1927$.

[23] Stepanova LV. Eigenvalue analysis for a crack in a power-law material. Computational Mathematics and Mathematical Physics 2009; 49 (8): $1332-1347$.

$3^{\text {rd }}$ International conference "Information Technology and Nanotechnology 2017" 
[24] Stepanova LV, Yakovleva EM. Mixed-mode loading of the cracked plate under plane stress conditions. PNRPU Mechanics Bulletin 2014; 3: 129-162.

[25] Torabi AR, Abedinasab SM. Brittle fracture in key-hole notches under mixed mode loading. Experimental study and theoretical predictions. Engineering Fracture Mechanics 2015; 134: 35-53.

[26] Stepanova LV, Igonin SA. Asymptotics of the near-crack-tip stress field of a growing fatigue crack in damaged materials: Numerical experiment and analytical solution. Numerical Analysis and Applications 2015; 8(2): 168-181.

[27] Kachanov LM. On rupture time under condition of creep. Izvestia Akademi Nauk SSSR Otd Tekhn Nauk 1958; 8: 26-31.

[28] Rabotnov YN. Creep problems in structure members. Amsterdam: North-Holland, 1969.

[29] Vesely V, Frantik P. Reconstruction of a fracture process zone during tensile failure of quasi-brittle materials. Applied and Computational Mechanics 2010; 4: $237-250$.

[30] Galouei M, Fakhimi A. Size effect, material ductility and shape of fracture process zone in quasi-brittle materials. Computers and Geotechnics 2015; 65: $126-135$.

[31] Frantik P, Vesely V, Kersner Z. Parallelization of lattice modelling for estimation of fracture process zone extent in cementitious composites. Advances in Engineering Software 2013; 60-61: 48-57.

[32] Fakhimi A, Wan F. Descrete element modeling of the process zone shape in mode I fracture at peak load and in post-peak regime. International Journal of Rock Mechanics \& Mining Sciences 2016; 85: 119-128.

[33] Wei MD, Dai F, Xu NW, Zhao T, Xia KW. Experimental and numerical study on the fracture process zone and fracture toughness determination for ISRMsuggested semi-circular bend rock specimen. Engineering Fracture Mechanics 2016; 154: 43-56. 